\title{
Biotecnologie avanzate per il trattamento sostenibile delle acque reflue
}

\author{
Alfonso Ronca ${ }^{1}$ \\ ${ }^{1}$ Affiliation not available
}

\begin{abstract}
Il trattamento delle acque reflue risulta prioritario nell'ottica di uno sviluppo sostenibile, in quanto l'utilizzo delle moderne biotecnologie può produrre effluenti di elevata qualità, pronti allo sversamento e/o al riutilizzo.

In quest'ottica, i bioreattori a membrana (MBR) risultano una tecnologia promettente. Il riscontro maggiormente osservato dalla comunità scientifica riguarda la qualità dell'effluente dopo il trattamento con MBR: esso risulta di qualità elevata e sostanzialmente disinfettato. D'altro canto, la problematica del fouling va a ridurre il loro utilizzo a scala reale.

Recenti studi hanno fatto luce su vari scenari volti al miglioramento degli MBR per mitigare il fenomeno del fouling e per migliorare le performance depurative.

Le tecnologie che si analizzano in questo articolo sono le membrane dinamiche autoformanti (SFDM), l'applicazione di processi elettrochimici al trattamento delle acque reflue (eMBR) e l'inoculazione di microalghe con fanghi attivi nel bioreattore (AS-MBR).
\end{abstract}

\section{Introduzione}

I sistemi MBR presentano numerosi vantaggi rispetto ai trattamenti convenzionali a fanghi attivi per la depurazione delle acque reflue. Il riscontro maggiormente osservato dalla comunità scientifi- 
ca riguarda l'effluente prodotto, il quale risulta di qualità elevata e sostanzialmente di disinfettato, pronto allo sversamento o al riutilizzo per scopi agricoli e/o industriali ${ }^{1,2}$. Questo aspetto risulta compatibile nell'ottica europea del "Green Deal”, dove si affronta la necessità di rivedere le politiche di gestione dell'energia e delle acque per evitare gli sprechi e promuovere comportamenti, come il riutilizzo delle acque, volti alla sostenibilità ambientale ${ }^{3}$. Utilizzando tale soluzione impiantistica, inoltre, il ciclo di trattamento di depurazione risulta semplificato (Fig. 1) e, dunque, vengono ridotti gli ingombri planimetrici delle vasche ${ }^{4}$.

Aspetto di ulteriori ricerche ed analisi riguardo la tecnologia MBR è il fouling: l'adesione di biomassa sulle superfici della membrana ne ostruisce i pori, rendendo i sistemi poco performanti a livello depurativo ed, inoltre, economicamente non sostenibili. Tale problematica rende necessarie azioni volte al controllo del fouling ${ }^{5}$, come il rilassamento o il lavaggio frequente con aria del modulo della membrana: ciò porta ad un aumento dei costi per l'areazione. Se il fouling è irrecuperabile, ossia non rimovibile con le azioni di pulizia convenzionali, bisogna sostituire l'intero modulo, operazione assai costosa ${ }^{6,7}$. I principali precursori del fouling sono stati individuati come le Sostanze Polimeriche Extracellulari (EPS), i Prodotti Microbiche Solubili (SMP) e le Particelle Esopolimetiche Trasparenti (TEP) ${ }^{8}$. Le prime due consistono i macromolecole sotto forma di carboidrati e proteine, mentre le ultime sono caratterizzate da alta viscosità e sono frequenti in ambiente marino $9,10,11$.

\section{Biotecnologie avanzate applicate ai bioreattori a membrana}

Ad oggi, sono state studiate varie innovative biotecnologie che possono andare a mitigare il fenomeno del fouling in maniera efficiente. La più recente riguarda l'inoculazione di alghe con fanghi attivi (Algae Sludge - Membrane BioReactor), volta a mitigare il fouling, ma che influisce positivamente anche sulle performances depurative. Le microalghe investigate appartengono alla specie "Chlorella Vulgaris" e, tramite illuminazione del bioreattore, esse svolgono processi foto- 
reattivi e forniscono ossigeno ai batteri aerobi eterotrofi per mineralizzare gli inquinanti organici, come nitriti, nitrati e fosfati ${ }^{13,14}$. C'è la possibilità che possa crescere uno stato di fouling dovuto alle alghe soprattutto perché esse generano TEP ${ }^{15}$, ma comunque facilmente removibile tramite contro-lavaggio con acqua/gas e rilassamento, ${ }^{16}$.

Un'altra investigazione consiste nell'applicare il campo elettrico tramite l'inserimento di elettrodi nei bioreattori a membrana (electro-Membrane BioReactor). I processi elettrochimici si manifestano con elettrocoagulazione, elettroforesi ed elettroosmosi (Fig. ??), i quali modificano le caratteristiche del fango, andando a migliorare le performance depurative e ridurre il fouling ${ }^{17,18,19}$. Ciò soprattutto grazie all'ossidazione dell' anodo che, in base al materiale di cui è formato, libera ioni $\mathrm{Al}^{3+} \mathrm{o} \mathrm{Fe}^{2+}$ che, legandosi al fango, fungono da agente coagulante ${ }^{20,21}$. I fiocchi, allora, divengono più pesanti e decantano, in modo da non aderire alla membrana. Inoltre, vari studi hanno fatto luce sull'abbattimento dei metalli pesanti e dei contaminanti emergenti ${ }^{22,23}$, tra cui i composti farmaceutici, come Diclofenac (DCF), carbamazepine (CBZ) and amoxicillin (AMX) ${ }^{21,24}$, grazie ai processi elettrochimici: la loro rimozione risulta ottimale con l'applicazione continua del campo elettrico, ma, per ragioni pratiche, si preferisce un'applicazione discontinua per evitare consumi energetici troppo elevati ${ }^{25,26}$.Uno dei principali svantaggi riguarda l'attività batterica: un campo elettrico, superiore a $2.5 \mathrm{~A} / \mathrm{m}^{2}$, risulta troppo intenso e va ad inibire l'attività batterica, soprattutto quella dei batteri nitrificanti, e a danneggiare le cellule batteriche, portando ad un aumento dei SMP ${ }^{27,17}$. Sotto questo aspetto, sono vari i parametri che influenzano i processi elettrochimici, come il $\mathrm{pH}$, la densità di corrente, la temperatura, il tempo di esposizione alla corrente, per cui, quando si intende utilizzare gli eMBR, bisogna fare attenzione alle varie scelte progettuali.

Al fine di ridurre i costi legati all'adozione degli MBR, si può optare per l'utilizzo delle membrane dinamiche autoformanti (Self Forming Dynamic Membrane), in quanto più economiche rispetto alle membrane convenzionali ${ }^{28}$. Esse sono composte da un materiale di supporto di vario genere (nylon, TNT, dacron...), in configurazione piana o tubolare, avente porosità da 10 a 200 micron. 
Tramite il processo di filtrazione si deposita la biomassa su tale materiale di supporto e si formano degli strati batterici: il cake layer ed il gel layer ${ }^{29,30,31}$. Questi riducono la porosità del supporto, andando a migliorare il processo di filtrazione. Quando la membrana dinamica è formata, ossia siamo in condizioni operative stabili, la qualità dell'effluente è paragonabile a quella di un effluente generato da membrane convenzionali. Dunque, il principale svantaggio nell'uso di tali membrane riguarda la scarsa qualità del permeato all'inizio del processo di filtrazione, ossia quando la membrana dinamica non è ancora formata.

\section{Risultati e prospettive future}

I risultati, di seguito riportati, riguardano un confronto tra impianti a scala di laboratorio ove vi erano implementate le biotecnologie precedentemente discusse. In sintesi, si confrontano le performance depurative e la mitigazione del fouling di:

- MBR covenzinale a fibre cave;

- e-MBR convenzionale a fibre cave con un intensità di corrente pari a $0.5 \mathrm{~mA} / \mathrm{cm}^{2}$;

- eAAS-SFDMBR con membrana dinamica autoformante (Alive Membrane ${ }^{\circledR},{ }^{32}$ ), inoculo di fanghi alghe pari a 5:1 ed un intensità di corrente pari a $0.5 \mathrm{~mA} / \mathrm{cm}^{2}$;

- AAS-SFDMBR con membrana dinamica autoformante ( Alive Membrane ${ }^{\circledR},{ }^{32}$ ) ed inoculo di fanghi alghe pari a 5:1.

Andando ad analizzare i sistemi con membrana dinamica autoformante, un parametro chiave nel trattamento del refluo sintetico è il tempo di formazione della membrana dinamica. Viene ricavato andando ad analizzare l'andamento della pressione transmembrana (TMP) e la torbidità degli effluenti. Si assume che la membrana è formata quando la pressione inizia ad aumentare, segno che la biomassa è adesa al materiale di supporto, e quando la torbidità scende sotto i 5 NTU ${ }^{30,31}$. I successivi depositi sulla membrana aumentano la resistenza alla filtrazione e quindi vi è la necessità di 
pulire il modulo della membrana ${ }^{33}$. Nel caso in esame, si è osservata una rapida diminuzione del valore della torbidità nell'effluente dell'eAAS-SFDMBR e, contestualmente, un aumento di TMP nelle prime ore di filtrazione. Si assume che il tempo di filtrazione per tale reattore sia risultato $25 \pm 5$ ore. Ancora, la TMP si è mantenuta costante durante tutto il periodo operativo, il che potrebbe portare tale soluzione impiantistica ad avere una durevole vita operativa. Per quanto riguarda l'AAS-SFDMBR, le tempistiche sono risultate differenti. Innanzitutto, la diminuzione della torbidità è risultata più lenta, così come il suo andamento durante la sperimentazione: infatti, la torbidità dell' effluente dell'eAAS-SFDMBR si è tenuto sotto i 0.6 NTU quasi sempre, a differenza dell'altro effluente i cui valori sono oscillati da 0.5 a 1.5 NTU. Inoltre, la TMP dell'AAS-SFDMBR, dopo un leggero aumento iniziale, segno di formazione della membrana, subisce un repentino aumento dopo circa 450 ore di sperimentazione, implicando una maggiore resistenza alla filtrazione. Si assume, per l'AAS-SFDMBR, un tempo di formazione pari a $80 \pm 20$ ore. Successivamente, si vanno ad analizzare le caratteristiche delle miscele areate tramite l'osservazione dei Solidi Sospesi Totali e Volatili e l'andamento della Clorofilla-a, indicatore della presenza di alghe. In generale, la differenza tra le caratteristiche delle due miscele areate è evidente, anche tramite la prova del cono imhoff (Fig. 2). I risultati, infatti, confermano una maggiore presenza di solidi nel reattore eAAS-SFDMBR, caratterizzati da maggiore sedimentabilità e omogeneità, mentre nel reattore AAS-SFDMBR troviamo meno solidi, i quali sono perlopiù filamentosi, e una maggiore quantità di alghe, denotate anche da un surnatante verdastro. Queste differenze risiedono nell'influenza positiva del campo elettrico, il quale è stato dimostrato avere effetti positivi sulla sedimentabilità del fango 34,26

Per quanto riguarda le performance depurative e la presenza dei precursori del fouling, i risultati si sono dimostrati ottimi, e confrontabili con sistemi MBR convenzionali. Infatti, i risultati ottenuti dai sistemi a membrana dinamica autoformante possono essere confrontati con la sperimentazione $\mathrm{di}^{8}$. La presenza delle alghe riduce la presenza delle EPS e dei SMP di più del $50 \%$ rispetto al sistema MBR convenzionale, ma la presenza dei processi elettrochimici aumenta ancora tale ridu- 
zione. Difatti, in tale ambito, l'eAAS-SFDMBR presenta concentrazioni bassissime di EPS e SMP, anche del 70\% inferiore dell'MBR convenzionale e del 50\% inferiore rispetto l'eMBR. Riguardo le TEP, invece, la loro concentrazione è molto alta sia nell' AAS-SFDMBR che nell'eAAS-SFDMBR rispetto sia all'MBR convenzionale che all'eMBR. Ciò è dovuto alle alghe, le quali favoriscono la proliferazione di TEP ${ }^{15}$. D'altro canto, il campo elettrico risulta un ottimo mezzo per ridurre la proliferazione di TEP, infatti nell'eAAS-SFDMBR abbiamo una riduzione di più del 50\% inferiore rispetto all'AAS-SFDMBR.

In merito alle performance depurative, si analizza l'abbattimento di COD (99.1\% per l'eAASSFDMBR e 97.10\% per l'AAS-SFDMBR) e di azoto ammoniacale (94.86\% per l'eAAS-SFDMBR e $92.77 \%$ per l'AAS-SFDMBR). La riduzione di COD è ottima in tutti i sistemi MBR analizzati. Inoltre, si assiste ad un ottimo processo di nitrificazione grazie all'inoculazione di alghe, ulteriormente migliorato dai processi elettrochimici. Difatti, sia rispetto al sistema MBR che all'eMBR, la rimozione di azoto ammoniacale risulta quasi completa grazie alla presenza delle alghe.

\section{Conclusione}

In conclusione, la connessione del campo elettrico con le alghe, unito un processo di filtrazione tramite membrana dinamica autoformante, risulta la soluzione più efficiente rispetto la presenza delle alghe. I risultati ottenuti dall'eAAS-SFDMBR si rivelano una tematica da approfondire ulteriormente, in quanto altamente promettente per il trattamento avanzato e sostenibile delle acque reflue. Lo studio condotto ha permesso di ottenere ottimi risultati, promettenti per lo sviluppo di biotecnologie altamente innovative volte alla depurazione sostenibile delle acque reflue e riutilizzo della risorsa. Nonostante i risultati positivi occorrono altri approfondimenti al fine di poter comprendere in fondo tutti i meccanismi che prendono parte al processo di depurazione e verificando la fattibilità tecnica ed economica per lo sviluppo scala reale di questa importantissima innovazione 
biotecnologica e scientifica.

\section{References}

1.Vassallo, V. Trattamento avanzato delle acque reflue urbane mediante bioreattori a membrane autoformanti per il controllo dei contaminanti emergenti . (2020) doi:10.22541/au.158921776.60948769.

2.Borea, L. et al.. Wastewater treatment by membrane ultrafiltration enhanced with ultrasound: Effect of membrane flux and ultrasonic frequency. Ultrasonics 83, 42-47 (2018).

3.Naddeo, V. \& Korshin, G. Water energy and waste: The great European deal for the environment. Science of The Total Environment 764, 142911 (2021).

4.Judd, S. The status of membrane bioreactor technology. Trends in Biotechnology 26, 109-116 (2008).

5.Fortunato, L., Ranieri, L., Naddeo, V. \& Leiknes, T. O. Fouling control in a gravity-driven membrane (GDM) bioreactor treating primary wastewater by using relaxation and/or air scouring. Journal of Membrane Science 610, 118261 (2020).

6.Drews, A. Membrane fouling in membrane bioreactors-Characterisation contradictions, cause and cures. Journal of Membrane Science 363, 1-28 (2010).

7.Naddeo, V., Belgiorno, V., Borea, L., Secondes, M. F. N. \& Ballesteros, F. Control of fouling formation in membrane ultrafiltration by ultrasound irradiation. Environmental Technology 36, 1299-1307 (2014).

8.Borea, L. et al.. Are pharmaceuticals removal and membrane fouling in electromembrane bioreactor affected by current density?. Science of The Total Environment 692, 732-740 (2019). 
9.Villacorte, L. O., Kennedy, M. D., Amy, G. L. \& Schippers, J. C. The fate of Transparent Exopolymer Particles (TEP) in integrated membrane systems: Removal through pre-treatment processes and deposition on reverse osmosis membranes. Water Research 43, 5039-5052 (2009).

10.Lin, H. et al.. A critical review of extracellular polymeric substances (EPSs) in membrane bioreactors: Characteristics roles in membrane fouling and control strategies. Journal of Membrane Science 460, 110-125 (2014).

11.Teng, J. et al.. Effects of molecular weight distribution of soluble microbial products (SMPs) on membrane fouling in a membrane bioreactor (MBR): Novel mechanistic insights. Chemosphere 248, 126013 (2020).

12.Naranjo, D. A. C. Procesos MBR y MBR con electrocoagulación (SMEBR): estudio en planta piloto de la influencia de la electrocoagulación en el ensuciamiento de la membrana. (Universidad de Alicante, 2019).

13.Yang, J. et al.. Potential of wastewater treatment using a concentrated and suspended algalbacterial consortium in a photo membrane bioreactor. Chemical Engineering Journal 335, 154-160 (2018).

14.Sun, L. et al. A novel symbiotic system combining algae and sludge membrane bioreactor technology for wastewater treatment and membrane fouling mitigation: Performance and mechanism. Chemical Engineering Journal 344, 246-253 (2018).

15.Corpuz, M. V. A. et al. Wastewater treatment and fouling control in an electro algaeactivated sludge membrane bioreactor. Science of The Total Environment 147475 (2021) doi:10.1016/j.scitotenv.2021.147475.

16.Fortunato, L., Lamprea, A. F. \& Leiknes, T. O. Evaluation of membrane fouling mitigation strategies in an algal membrane photobioreactor (AMPBR) treating secondary wastewater effluent. Science of The Total Environment 708, 134548 (2020). 
17.Ensano, B. M. B. et al.. Combination of Electrochemical Processes with Membrane Bioreactors for Wastewater Treatment and Fouling Control: A Review. Frontiers in Environmental Science 4, (2016).

18.Borea, L., Naddeo, V. \& Belgiorno, V. Application of electrochemical processes to membrane bioreactors for improving nutrient removal and fouling control. Environmental Science and Pollution Research 24, 321-333 (2016).

19.Jallouli, S. et al.. Efficient and sustainable treatment of tannery wastewater by a sequential electrocoagulation-UV photolytic process. Journal of Water Process Engineering 38, 101642 (2020).

20.Naranjo, D. A. C. Procesos MBR y MBR con electrocoagulacion (SMEBR): estudio en planta piloto de la influencia de la electrocoagulacion en el ensuciamiento de la membrana. (Universidad de Alicante, 2019).

21.Ensano, B. et al. Removal of Pharmaceuticals from Wastewater by Intermittent Electrocoagulation. Water 9, 85 (2017).

22.Prado, M. et al.. Removal of emerging contaminant and fouling control in membrane bioreactors by combined ozonation and sonolysis. International Biodeterioration \& Biodegradation 119 , 577-586 (2017).

23.Ibrahim, Y., Abdulkarem, E., Naddeo, V., Banat, F. \& Hasan, S. W. Synthesis of super hydrophilic cellulose-alpha zirconium phosphate ion exchange membrane via surface coating for the removal of heavy metals from wastewater. Science of The Total Environment 690, 167-180 (2019). 24.Naddeo, V., Ricco, D., Scannapieco, D. \& Belgiorno, V. Degradation of Antibiotics in Wastewater during Sonolysis Ozonation, and Their Simultaneous Application: Operating Conditions Effects and Processes Evaluation. International Journal of Photoenergy 2012, 1-7 (2012). 
25.Ensano, B. M. B. et al.. Applicability of the electrocoagulation process in treating real municipal wastewater containing pharmaceutical active compounds. Journal of Hazardous Materials 361, 367-373 (2019).

26.Borea, L. et al. . Microbial fuel cell technology as a downstream process of a membrane bioreactor for sludge reduction. Chemical Engineering Journal 326, 222-230 (2017).

27.Zhang, J. et al.. Low-voltage electric field applied into MBR for fouling suppression: Performance and mechanisms. Chemical Engineering Journal 273, 223-230 (2015).

28.Millanar-Marfa, J. M. J. et al.. Self-forming Dynamic Membranes for Wastewater Treatment. Separation \& Purification Reviews 1-17 (2021) doi:10.1080/15422119.2021.1887223.

29.Ersahin, M. E. et al.. A review on dynamic membrane filtration: Materials applications and future perspectives. Bioresource Technology 122, 196-206 (2012).

30.Rezvani, F., Mehrnia, M. R. \& Poostchi, A. A. Optimal operating strategies of SFDM formation for MBR application. Separation and Purification Technology 124, 124-133 (2014).

31.Mohan, S. M. \& Nagalakshmi, S. A review on aerobic self-forming dynamic membrane bioreactor: Formation performance, fouling and cleaning. Journal of Water Process Engineering 37, $101541(2020)$.

32.Naddeo, V., Belgiorno, V., Borea, L., Castrogiovanni, F. \& Napodano, P. UNITÀ, DISPOSITIVO, METODO E SISTEMA DI FILTRAZIONE PER IL TRATTAMENTO AVANZATO E SOSTENIBILE DI ACQUE REFLUE. Patent under evaluation.. (2020).

33.Yu, Z., Hu, Y., Dzakpasu, M. \& Wang, X. C. Thermodynamic prediction and experimental investigation of short-term dynamic membrane formation in dynamic membrane bioreactors: Effects of sludge properties. Journal of Environmental Sciences 77, 85-96 (2019).

34.ElNaker, N. A., Elektorowicz, M., Naddeo, V., Hasan, S. W. \& Yousef, A. F. Assessment of 
Microbial Community Structure and Function in Serially Passaged Wastewater Electro-Bioreactor Sludge: An Approach to Enhance Sludge Settleability. Scientific Reports 8, (2018).

35.Errami, M. \& Garner, H. A tale of two citations. Nature 451, 397-399 (2008). 


\section{Figure Captions}

Figure 1. Comparazione tra soluzioni impiantistiche per il trattamento delle acque reflue tra sistemi convenzionali (a) e sistemi MBR (b) (adattato da ${ }^{12}$ ).

Figure 2. Prova del cono imhoff tra eAAS-SFDMBR e AAS-SFDMBR 


\section{Figures}

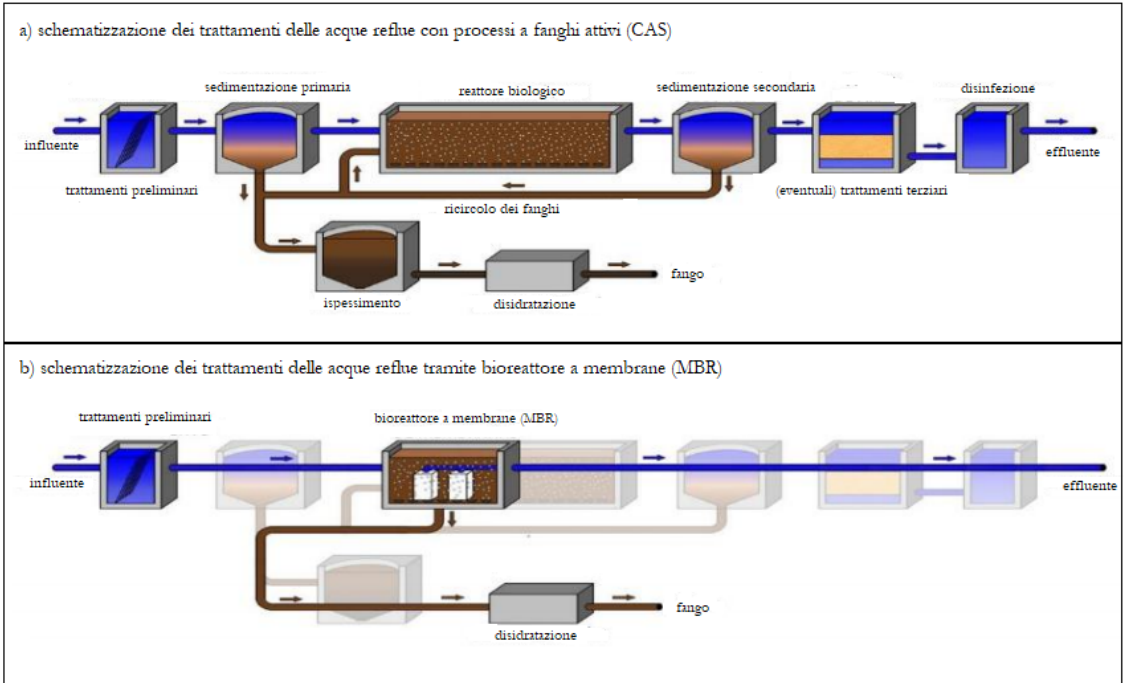

Figure 1: Comparazione tra soluzioni impiantistiche per il trattamento delle acque reflue tra sistemi convenzionali (a) e sistemi MBR (b) (adattato da ${ }^{12}$ ). 


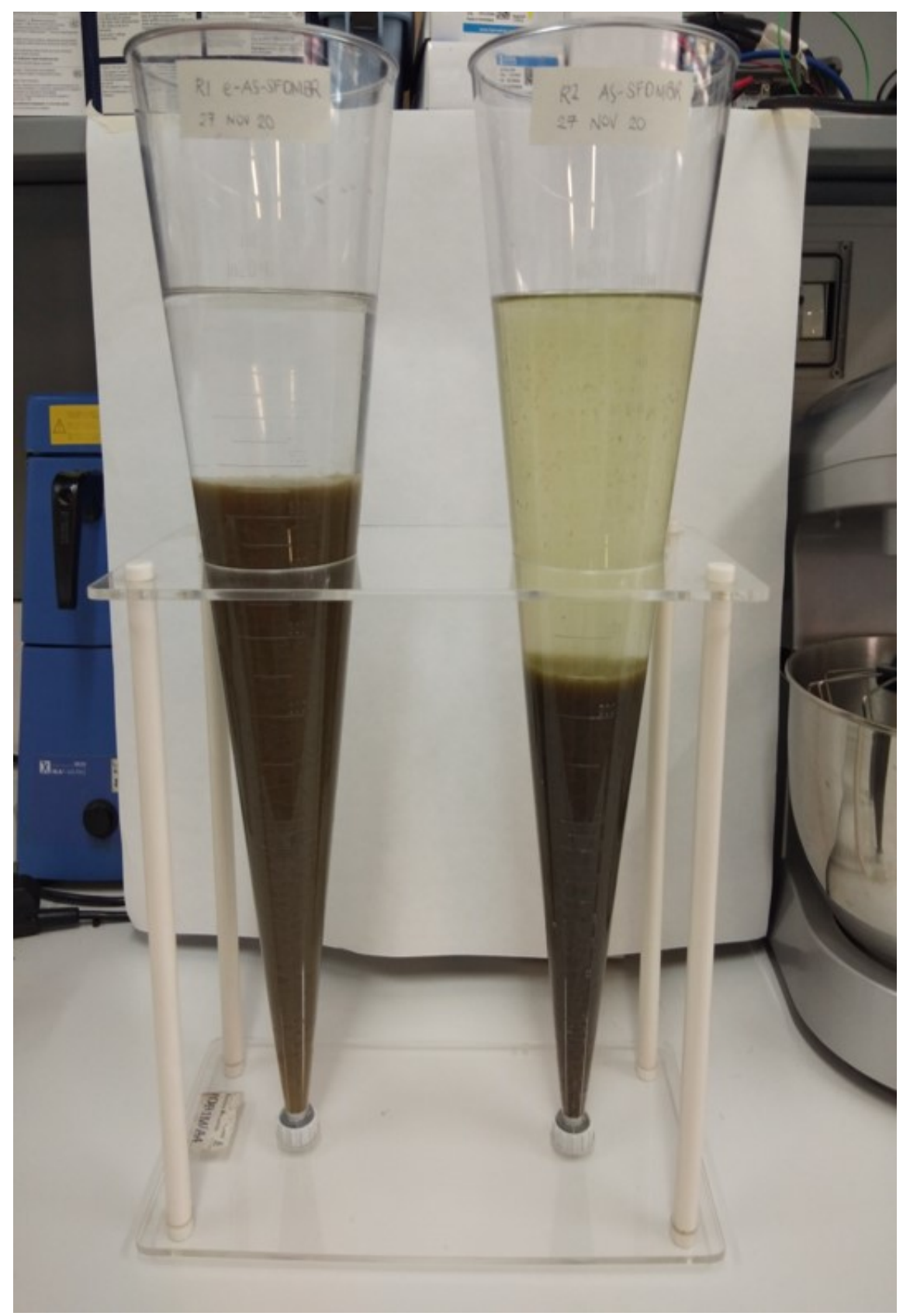

Figure 2: Prova del cono imhoff tra eAAS-SFDMBR e AAS-SFDMBR 